\title{
Einzelbesprechung \\ Person/Selbst
}

Stephen P. Turner, Cognitive Science and the Social. A Primer. New York/Abingdon: Routledge 2018, 226 S., kt., 28,60€

Besprochen von Dr. Judith H. Martens: Universität Wien, Institut für Philosophie, Wien, E-Mail: Judith.martens@univie.ac.at

https://doi.org/10.1515/srsr-2020-0073

Schlüsselwörter: Interdisziplinarität, Kognitionswissenschaft, Sozialtheorie, Lehre

The work under consideration addresses a fundamental problem in cognitive neuroscience and social science. Although both aim to explain and understand human action, their explanatory tools are so divergent that our theories are riddled with conceptual gaps. Both fields are moreover permeated by old-fashioned action theory and folk psychology, which explain and understand action in terms of mind-reading and attributing beliefs, desires, and intentions to others. While these theories may work pragmatically in navigating our social world, they are increasingly questioned as a basis for our theorizing. These and other problems within both disciplines have long held back a much-needed multidisciplinary approach to human action from gaining traction. Turner's book aims to set it free.

In what follows I assess the book based on the key argumentative steps in Turner's analysis, rather than providing a chapter-by-chapter overview.

As is well known, ordinary people rely on folk-psychological notions to make sense of themselves and others. Yet we know that when people interact, rather than talk about or describe their interacting, they don't necessarily rely on such folk-psychological notions. The social sciences nevertheless use this model of human agency in their theorizing. Perhaps surprisingly, the model also has the cognitive sciences under its spell. Their interest lies with cognitive capacities, which in themselves are often non-agentic, tacit, implicit, unconscious, etc. Nevertheless, cognitive scientists tend to interpret such capacities in folk-psychological terms.

Thus, cognitive and social scientists are tied up in an implicit web of folkpsychological explanations of cognitive capacities and human agency. Both are therefore stuck in what Turner calls the Verstehen bubble. Although he remains somewhat unclear on the details of this bubble, I take it to mean something like 
this: Our understanding of ourselves and others, both in ordinary interaction as well as scientifically, is limited because there is no clear way of mapping quantitative descriptions of cognitive capacities on implicit and explicit qualitative notions of understanding human agency. It is crucial, then, Turner argues, that scientists in both domains account for this in their theorizing.

Unfortunately, Turner argues, theorists have too often taken connections between the quantitative research and the interpretation for granted. They regularly fall prey to "must be so" analyses and ignore how the complexities flowing from the incommensurable structures of the tacit and the explicit problematize giving an account of the nature of our cognitive capacities and their relation to our selfunderstanding. This is further aggravated by cultural variation.

This is the book's main idea. I believe it is a valuable critique to standard approaches. But how does Turner support his critique? And what are his alternatives? I first turn to his critique.

\section{Perspectives on Cognition}

Turner takes a critical stance towards "the standard approach" (SA) in cognitive science: computationalism (information processing in a physical symbol system, or computation over representation).

Turner points at four problems that the SA face. The SA models cognitive computation on explicit reasoning. If we begin with 'reasoning with concepts' as a cognitive capacity, we end up with a picture where the brain processes representations through rules embedded in the brain to produce new representation. This means, however, that the SA treats representations as simultaneously causal and semantic, and thus governed by logic.

1. The concepts of representation used in the computational accounts themselves, however, are not semantic or meaningful in the required sense.

2. Furthermore, such computation is expensive, it causes cognitive overload.

To circumvent cognitive overload, cognitive science ascribes "architectural" features to the mind's capacities: fixed computational modules which are universal and innate. Communication and mutual understanding are thought to be possible because of these shared modules and mechanisms. There are two problems with this solution:

3. Universal modules require a long process of natural selection.

4. Universal modules sit uneasy with cultural variance. Social skills are culturally diverse and acquired. 
Turner then applies these critiques to our understanding of several social phenomena that cognitive science has modelled, including explaining and understanding action, selves, and persons.

\section{Exploring a New Approach}

Turner combines 4E cognition and the idea of the pervasive social as a fresh perspective to modelling and understanding cognitive capacities. This approach focuses on the active, acting subject. It understands cognitive processes as elements in a changing system of relations between the environment and the subject.

$4 \mathrm{E}$ cognition and the idea of the pervasive social deny (massive) modularism. For Turner's approach to be a credible alternative, it must, crucially, be able to explain rapid learning (for this is a major strength of modularism). Turner proposes a connectionist model of learning supplemented with facilitators that help speed up the learning process. This is compatible with predictive processing models. General expectations, rather than pre-existing concepts, fire up the learning engine, which takes the form of reducing signal noise, or irrelevance, in "the buzzing, blooming world”. His model allows for a better explanation of local differences in cognition while it allows for a shared background.

Turner introduces five building blocks that play an important role in his understanding of (social) cognition.

1. The presence of multiple, redundant pattern recognition systems, some of which are innate, others learned.

2. The tendency to over-attribute or over-categorize, i.e. to find patterns where there might be none.

3. A looping, feedback relation between innate and learned cognitive capacities that alters their basic capacities.

4. A social domain that influences the cognitive domain, in part through the types of patterns we learn to recognize.

5. "Thinking slow" occurs when two recognized patterns are incompatible.

Pattern recognition is the basic cognitive capacity in Turner's model. It is part of skilled performance and improves with embodied experience. Inference doesn't rely on a belief-desire structure, but is a "pattern completion inference within situated conceptualizations" (Barsalou in Turner).

Some pattern-recognition flows from universal capacities (e.g. empathy), others are largely socially structured. Thus, Turner holds, we have a basic capacity of interpretation, but the contents of interpretation are cultural artefacts and depend on our habitual modes of feeling and thinking. These habitual modes are 
what makes interpretation intelligible to us, as interpreters or audience, they allow us to understand actions.

\section{Solving the speed puzzle - incorporating the social into cognitive science}

In a chapter on affordances, scaffolding, and computational complexity, Turner explores how tacit acquired capacities can be as effortless and fast as modules. Pattern recognition again plays a key role, this time in the form of schemas. Schemas are "mental forms that can be repeated and shared, for example, to make up a culture" (131).

This understanding of skilled pattern recognition is based on bottom-up learning as well as top-down learning, Turner explains. Habits and patterns intertwine with narrativized public expressions. The sameness between minds is then accounted for through social interaction rather than by shared innate mechanisms. Pattern recognition and joint attention can speed up cognition, particularly when they become embedded in features of the situation. Thus, on Turner's account, routines, affordances, and objects can do a part of the mind's work.

\section{Selves, persons, and the social}

Turner defends a conception of the self that is not merely the product and expression of internal processes. Rather, (1) the self is socially constructed or narrativized and (2) the self and the social are intertwined on both the tacit and the explicit level. This implies, again, a shift from internal "architectural" explanations to explanations pointing at scaffolding, affordances, and external explanations of coherence.

\section{The Verstehen Bubble and the Pervasive Social}

Turner then connects the SA to cognitive science to the SA in social theory, the implicitly present 'Hobbesian social' which depends on the idea of "autonomous agents, and conceives of 'society' as the product of an arrangement between autonomous agents" (206). The problem with the standard model, Turner explains, is that it assumes a universal developmental process from which agents emerge. In it, culture is only a superficial overlay of the universal capacities all humans share. 
He finds solace in so-called 'interactionist' accounts, which are 'pervasively social' in their understanding of the development of cognitive capacities. They see almost everything, including the tacit, as socially distributed and - at least partly - the product of social learning, practice, and social processes themselves.

Following Mercier and Sperber's (2017) proposal, Turner suggests that metacognition and reasons are there for our interactions with others. Starting from the assumption of an overly active, pattern-recognizing brain, the idea is developed that aspects of culture are generated through a mismatch between the proper and the actual domain of cognitive modules, which as a side effect leads to the development of cultural ideas, practices, and artefacts. Such “errors”, when shared, are a valuable basis for the kinds of social prediction that are central to social interaction.

Patterns are used to find the most predictable and/or valuable options (of understanding and acting). Such predictions, especially over time, depend on joint attention and social inputs. Through our interactions, our socio-cultural practices hijack our more basic (emotional) perception of the world. Such practices are both external and internal to the individual.

\section{Some Further Reflections}

Turner's proposal has much to be commended and is based on a lucid, wide-ranging overview of the literature in several fields. His warnings regarding the pitfalls in understanding and integrating cognitive science in our theorizing of human action should be heeded by scholars in the field. His analysis of the problems of computationalism and his exposition of the 'pervasively social' alternative are particularly attractive.

On a more critical note, I felt that Turner sometimes unnecessarily remained stuck in old paradigms where I felt literature that could significantly contribute to his project was available but overlooked. Turner takes affordances, patterns, habits, and schemas as cognitive shortcuts. This, I believe, is counter-productive because it entails explicit and cognitive demanding cognition as the standard from which we take shortcuts. Something similar happens when Turner uses Dreyfus' ideas on skills as surpassing slow thinking. The recent debate on skilful action offers interesting suggestions that circumvent these standard, "shortcut" ways of thinking (e.g., Christensen/Sutton/McIlwain, 2016; Fridland, 2014). This type of work also builds on theories of attention, another key concept that could have benefitted Turner's integration of the pervasive social in his model (e.g., Wu, 2011; 2014).

The most glaring omission in Turner's integrative project, I felt, was research on core cognition. Particularly the way in which innate and learned capacities are 
understood in this literature could improve Turner's ideas on the hijacked basic capacities (e.g. Carey, 2009; Kinzler/Spelke, 2007; Spelke, 2000). A similar case could be made for recent literature that understands cognitive development from a more evolutionary perspective and explains changes in our cognitive systems by means of cultural evolution (e.g., Heyes, 2018; Sterelny, 2014).

These are merely suggestions that, for this particular reader, would have elevated an already thought-provoking 'primer' to a more substantive exposition of a new paradigm. We should look forward to Turner's continuation of his project.

\section{Literature}

Carey, S. The Origin of Concepts. Oxford University Press: Oxford, 2009.

Christensen, W.; Sutton, J.; Mcllwain, D. J. F. Cognition in Skilled Action: Meshed Control and the Varieties of Skill Experience. Mind \& Language 2016, 31, 37-66.

Fridland, E. They've Lost Control: Reflections on Skill. Synthese 2014, 191, 2729-2750.

Heyes, C. Cognitive Gadgets: The Cultural Evolution of Thinking; Harvard University Press: Harvard, 2018.

Kinzler, K. D.; Spelke, E. S. Core Systems in Human Cognition edited by C. von Hofsten and K. Rosander. Progress in Brain Research 2007, 164, 257-264.

Mercier, H.; Sperber, D. The Enigma of Reason: A New Theory of Human Understanding; Harvard University Press: Cambridge Massachusetts, 2017.

Spelke, E. S. Core Knowledge. The American Psychologist 2000, 55, 1233-1243.

Sterelny, K. The Evolved Apprentice: How Evolution Made Humans Unique; MIT Press: Cambridge, Ma., 2014.

Wu, W. Confronting Many-Many Problems: Attention and Agentive Control. Noûs 2011, 45, 50-76. Wu, W. Attention; Routledge: New York/Abingdon, 2014. 